\title{
Development of antenna arrays with a beam width 33 degrees at the level of half power
}

\author{
Viacheslav Rudakov ${ }^{1, *}$ and Victor Sledkov ${ }^{2}$ \\ ${ }^{1}$ South Federal University, Zorge st. 5, Rostov-on-Don, 344090, Russian Federation \\ ${ }^{2}$ Guandong Sigtenna Communication Technology Co., Ltd., Jin da Automobile logistic city, No. 4, \\ Yanwo village, Shipai Town, Dongguan, China
}

\begin{abstract}
A new design of the antenna array for mobile base stations providing two orthogonal polarizations in $1.71-2.17 \mathrm{GHz}$ frequency band is proposed. The antenna array includes 16 radiators, 12 power dividers and two phase shifters disposed on a metal reflector having dimensions $320 \times 1387 \mathrm{~mm}$. The developed antenna array contains less radiators and power dividers than other known antennas of this kind therefore one is cheaper for production. The fabricated sample of the proposed antenna array has return loss is better than $-18.5 \mathrm{~dB}$ and isolation better than $33 \mathrm{~dB}$. Beam width in the horizontal plane is equal $36 \pm 4$ degrees and side lobes level doesn't exceeding $-20 \mathrm{~dB}$. The antenna array provides gain $=20.5 \mathrm{dBi}$.
\end{abstract}

\section{Introduction}

In these days of high mobile phone usage, market needs huge quantity of dual polarized antennas each year therefore considerable work has been done in this field to create such antennas which are easy to be manufactured. The most of used in the past antenna arrays for base station create narrow beam at vertical plane and wide beam at horizontal plane to cover 120 degree sector. Now at some areas traffic through 120 degree sector is too big for one base station therefore it is need to add the second base station and use one base station to cover 60 degree sector only. Known antenna arrays operating at 60 degree sector usually contain two radiators connected in parallel by power divider in each row to decrease beam width at horizontal plane. One example of common dual polarized antenna array providing 33-40 degrees half power beam width at horizontal plane is described by [1].

The present paper is devoted to research and improvement of the antenna array providing two orthogonal polarizations in the 60 degree sector. A beam of known antenna arrays is narrowed by installing two parallel-connected radiators at each row [1]. The main disadvantage of this design is that a pair of connected in parallel radiators creates a large frequency dependence of the beam width. At the lower operating frequency, a too wide beam illuminates adjacent sectors, and at a higher frequency, a too narrow beam doesn't provide a sufficient signal level at the edge of the sector and has a large side lobe falling into the neighboring sectors. The antennas of such kind contain twice more radiators and

\footnotetext{
*Corresponding author: ruva240187@yandex.ru
} 
additional power dividers therefore ones are more complicated for manufacturing than antenna arrays providing 65 degrees half power beam width at horizontal plane.

\section{The design of the antenna array}

During development of antenna arrays for base stations, the following basic requirements must be taken into account: a high level of gain, stability of the beam width in the horizontal plane, good matching and a low level of side lobes within the operating frequency band, high isolation between ports, compact and simple construction suitable for mass production. Starting from the prior art mentioned, it is therefore the first object of the present paper to create a dual-polarized antenna array providing 30-40 degree half power beam width and low side lobes at horizontal plane through at least $25 \%$ frequency band. The second object is to decrease quantity of radiators and power divider to simplify manufacturing of the antenna array.

The known antenna array for $1.7-2.17 \mathrm{GHz}$ frequency band containing 20 radiators placed in two columns and forming 10 rows wherein each row contains two radiators connected in parallel by a power divider is shown in Fig.1a. Distance between columns is adjusted to provide half power beam width less than 40 degree at the lowest frequency $1.71 \mathrm{GHz}$. Calculated patterns at horizontal plane of this known antenna array illustrated in Fig.1(b) show big difference of patterns at the lowest frequency $1.71 \mathrm{GHz}$ and the highest frequency $2.17 \mathrm{GHz}$ where side lobe level increases up to $15 \mathrm{~dB}$.

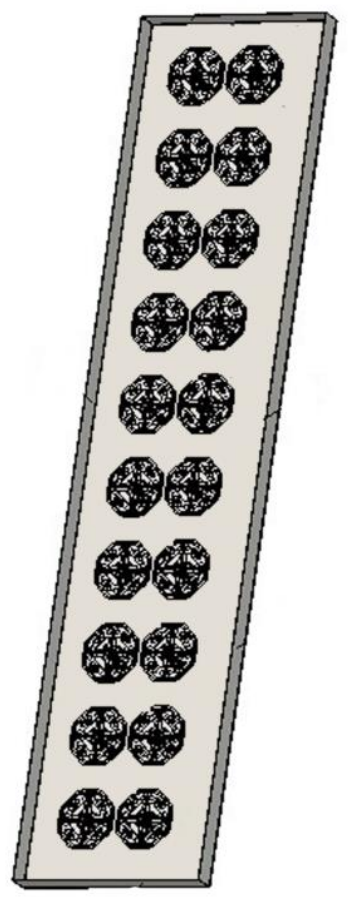

(a)

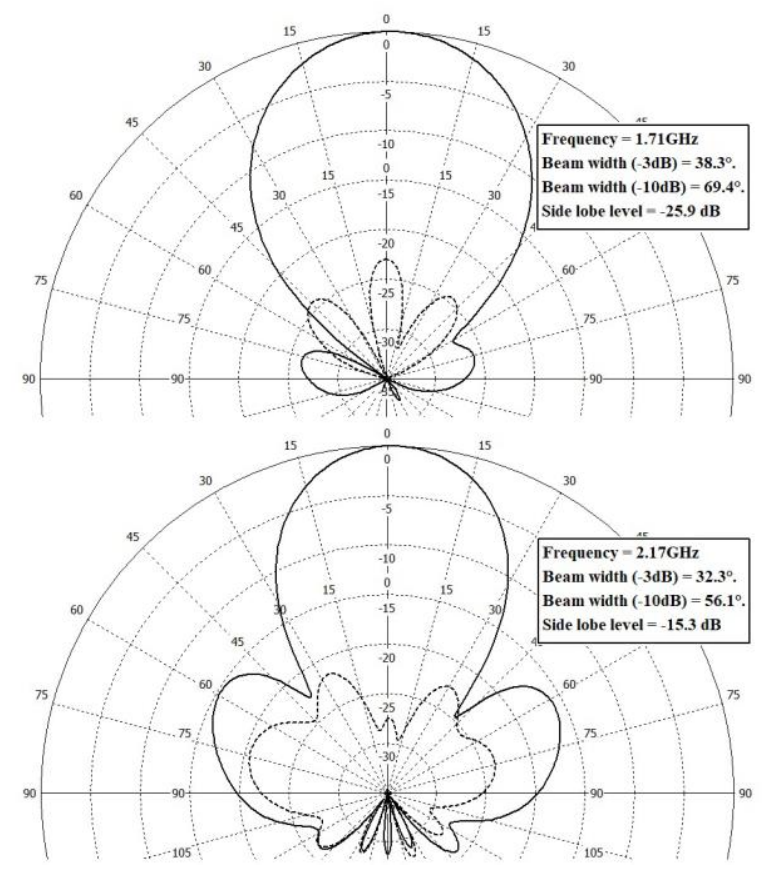

(b)

Fig. 1. (a) top view of the antenna array, (b) normalized radiation patterns.

As a result of the development the new structure was found that allows stabilize the beam width and at the same time reduce the number of radiators and power dividers compared with previously known designs $[1,2]$. 
The new structure provides the dual polarized antenna array containing $\mathrm{N}$ rows of the dual polarized radiators placed on a conductive reflector and feed by two beam forming networks having $\mathrm{N}$ outputs each and forming narrow beam at vertical plane. At least $\mathrm{N} / 2$ rows placed at the middle part of the antenna array contain two radiators connected in parallel by two power dividers fed by the beam forming networks. Other rows placed at top and bottom parts of the antenna array contain only one radiator directly connected to the beam forming networks. The frequency dependence of the beam width of the antenna array also is decreased because the beam width of single radiators depends on the frequency much less than the beam width of a pair of radiators. Single radiators radiate in direction of side lobes maximum signal with different phase in compare with radiation of a pair of radiators therefore partly suppress side lobes.

The conductive reflector contains side walls along longitudinal edges. Width of the reflector and high of bent up side walls adjusted to provide half power beam width less than 40 degree at the lowest frequency $1.71 \mathrm{GHz}$. Additional longitudinal side walls placed near radiators of the middle column change its radiation towards edges of the reflector therefore it is possible to adjust dimensions of additional longitudinal side walls and suppress side lobes of antenna arrays containing any quantity radiators and improve cross-polarization. The simplified electric circuit of the proposed antenna array containing 10 rows of the dual polarized radiators is shown in Fig. 2a. The perspective top view of this antenna array is shown in Fig. $2 b$.

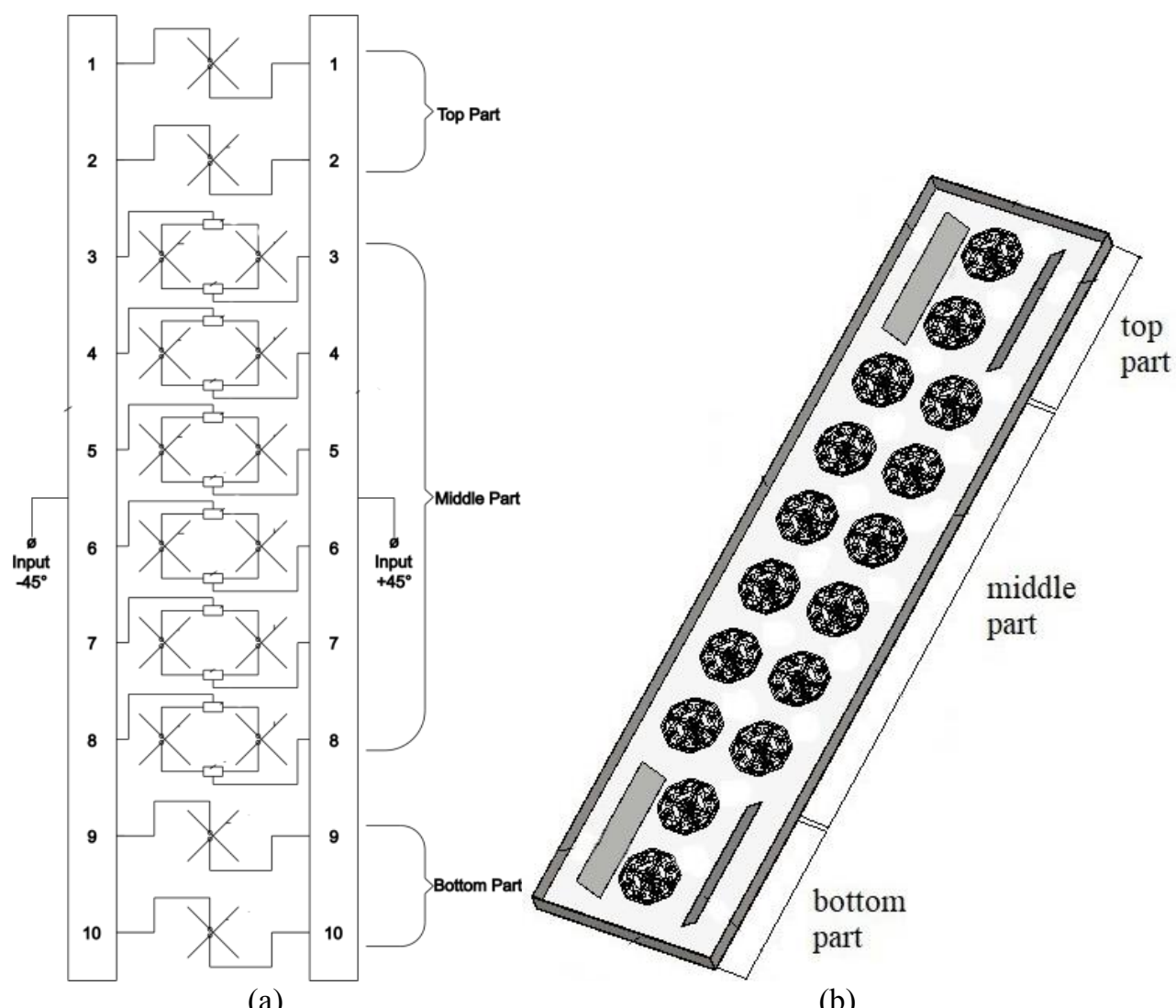

(a)

Fig. 2. The proposed antenna array (a) simplified electric circuit, (b) perspective top view. 


\section{Results of simulation and measurements}

The proposed antenna array was simulated and manufactured. Electrodynamic modeling and optimization are performed by the finite integration method in a specialized software package. The results of simulation and measurement are shown in Fig. 3.

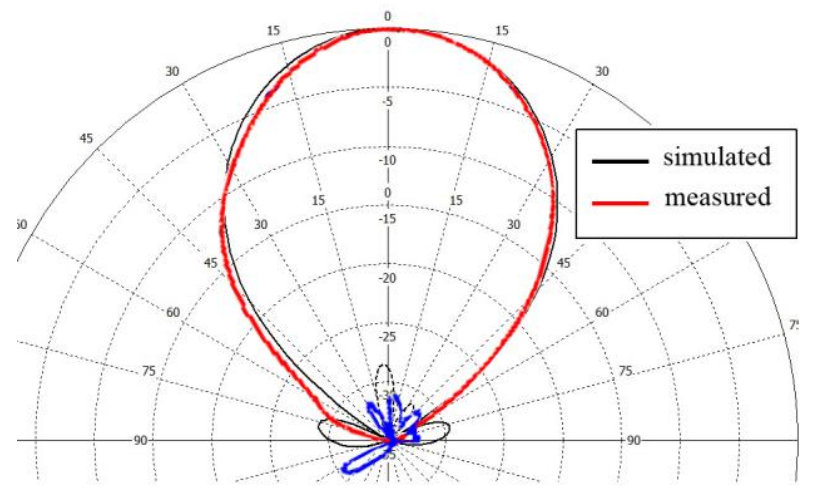

(a)

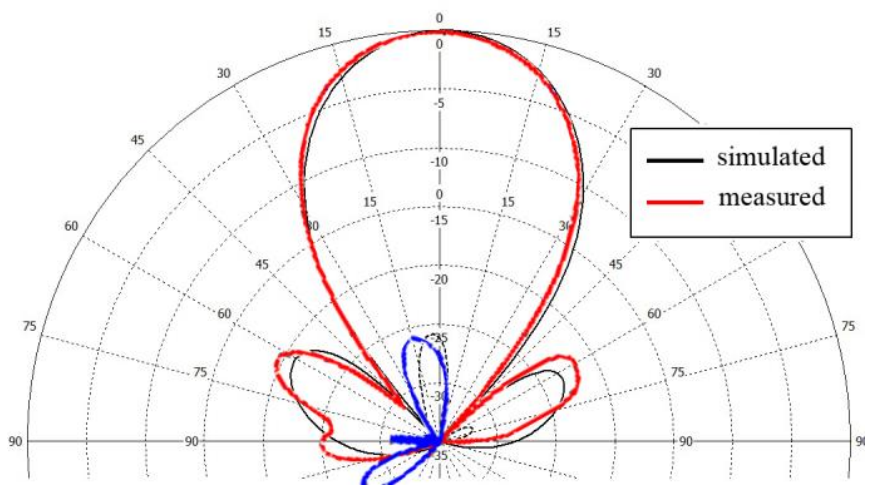

(b)

Fig. 3. Normalized radiation pattern at horizontal plane (a) $f=1.71 \mathrm{GHz}$, (b) $f=2.17 \mathrm{GHz}$.

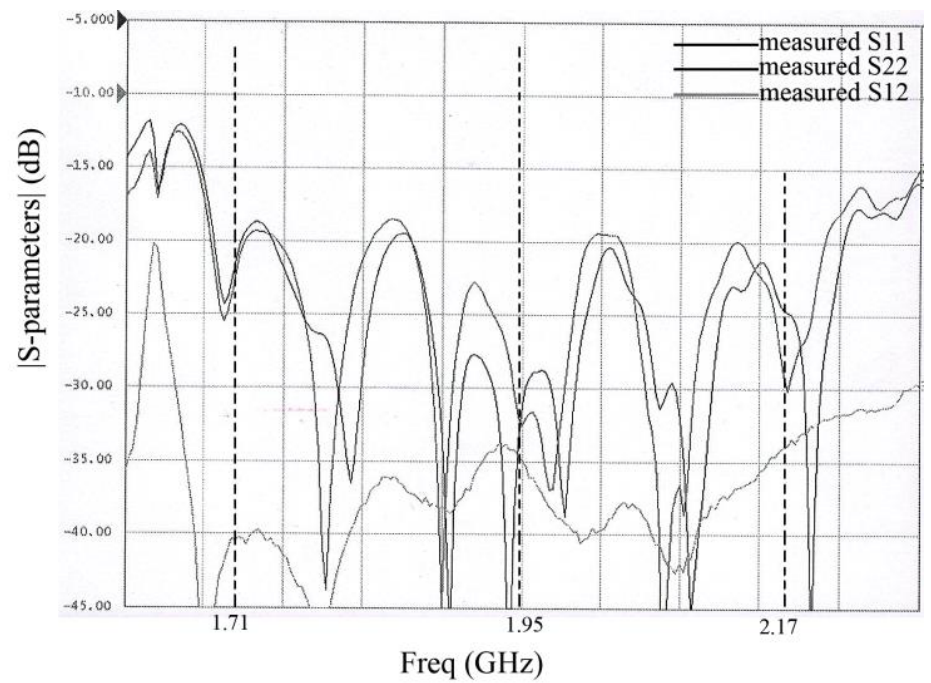

Fig.4. The measured return loss and isolation of the manufactured antenna array. 
According to the simulated model the horizontal beam width at half power is $38.3^{\circ}$, the side lobe level is $-29 \mathrm{~dB}$, and the gain better than $20.3 \mathrm{~dB}$ at $1.71 \mathrm{GHz}$. The horizontal beam width at the half power level is $31.9^{\circ}$, the side lobe level is $-20.9 \mathrm{~dB}$, and the gain is $21.6 \mathrm{~dB}$ at $2.17 \mathrm{GHz}$.

According to the experiment, the horizontal beam width at the half power level is $38.29^{\circ}$, the side lobe level is better than $-28.67 \mathrm{~dB}$, and the gain is $20.15 \mathrm{~dB}$ at $1.71 \mathrm{GHz}$. The horizontal beam width at half power is $34.5^{\circ}$, the side lobe level is $-19.5 \mathrm{~dB}$, and the gain is $21.54 \mathrm{~dB}$ at $2.17 \mathrm{GHz}$. Thus difference between results of simulation and measurement is quite small.

The measured $S$ parameters of the manufactured antenna array are shown in Fig. 4. The maximum measured return loss is $-18.5 \mathrm{~dB}$ and the isolation between two ports is better than $-33 \mathrm{~dB}$ within frequency band 1.71-2.17 GHz.

\section{Conclusion}

A new design of the dual-polarized antenna array is proposed in the paper, which provides a beam width in the horizontal plane at half power level of $36 \pm 4$ degrees within the frequency band $1.71-2.17 \mathrm{GHz}$. The gain is $20.5 \mathrm{~dB}$. Measured return loss of antenna array is better than $-18.5 \mathrm{~dB}$. A new design of the antenna arrays are better suit for modern base stations since provide small side lobes at horizontal plane and small upper side lobes at vertical plane. Side lobes do not exceed the level of -20dB. The dual-polarized antenna arrays according to the invention contains less radiators and power dividers than conventional antenna arrays therefore ones are easy for manufacturing. The developed antenna array, with 10 rows of radiators contains only 16 radiators and 12 power dividers in compare with conventional antenna array of the same length containing 20 radiators and 20 power dividers. The proposed construction quite simple for production and has compact overall dimensions $(176 \times 320 \times 1387 \mathrm{~mm})$.

\section{References}

1. Patent $C N 105846113 \mathrm{~A}$

2. Balanis C.A. Modern antenna handbook (USA 1938) 